\title{
A Role for Non-Rapid-Eye-Movement Sleep Homeostasis in Perceptual Learning
}

\author{
Daniel Aeschbach, ${ }^{1,2}$ Alex J. Cutler, ${ }^{1}$ and Joseph M. Ronda ${ }^{1,2}$ \\ ${ }^{1}$ Division of Sleep Medicine, Brigham and Women's Hospital, and 'Division of Sleep Medicine, Harvard Medical School, Boston, Massachusetts 02115
}

\begin{abstract}
Slow-wave activity (SWA; EEG power density in the $0.75-4.5 \mathrm{~Hz}$ range) in non-rapid-eye-movement (NREM) sleep is the primary marker of sleep homeostasis and thought to reflect sleep need. But it is unknown whether the generation of SWA itself serves a fundamental function. Previously, SWA has been implicated in brain plasticity and learning, yet the evidence for a causal role remains correlative. Here, we used acoustic slow-wave suppression to test whether overnight improvement in visual texture discrimination, a form of perceptual learning, directly depends on SWA during sleep. Two groups of subjects were trained on a texture discrimination task (TDT) after baseline sleep, and were tested $24 \mathrm{~h}$ later, after a $4 \mathrm{~h}$ experimental (EX) sleep episode (with or without SWA suppression), and again after a night of recovery sleep. In the suppression group, SWA during EX sleep was reduced by $30 \%$ compared with the control group, whereas total sleep time and REM sleep were not affected. Texture discrimination improved after EX sleep in the control group but not in the suppression group. Moreover, overnight improvement in TDT performance correlated with EEG power density during NREM sleep in the frequency range of SWA (maximum $r=0.75$ at $0.75-1.0 \mathrm{~Hz}$ ) over brain areas involved in TDT learning. We conclude that SWA is an important determinant of sleep-dependent gains in perceptual performance, a finding that directly implicates processes of sleep homeostasis in learning.
\end{abstract}

Key words: sleep homeostasis; slow-wave activity; slow-wave suppression; perceptual learning; texture discrimination; plasticity

\section{Introduction}

Electroencephalographic (EEG) slow waves are the hallmark of deep non-rapid-eye-movement (NREM) sleep in humans and other mammals. These waves arise from the slow oscillation $(<1$ $\mathrm{Hz}$ ) of the membrane potential of millions of cortical neurons between phases of depolarization and hyperpolarization (Steriade et al., 1993). Slow waves have attracted much scientific interest because their prevalence and amplitude, typically quantified by spectral analysis as slow-wave activity (SWA) (power density in the $0.75-4.5 \mathrm{~Hz}$ range), appear to reflect sleep need (Borbély, 1982; Daan et al., 1984). SWA increases in proportion to an individual's previous time awake, and it decreases during sleep. It is therefore thought to be a manifestation of a homeostatic sleep regulatory process. However, it is not known whether SWA is a mere marker of the homeostatic process or whether it directly serves a fundamental function.

Sleep has been linked to brain plasticity. In developing ani-

Received Sept. 20, 2007; revised Jan. 29, 2008; accepted Jan. 30, 2008.

This work was supported by awards from National Alliance for Research on Schizophrenia and Depression and the Milton Fund of Harvard University (D.A.), as well as by National Institutes of Health Grant NCRR-GCRC-M01-RR02635 (Brigham and Women's Hospital). We thank Dr. Nayantara Santhi for comments on this manuscript and Dr. Wei Wang for statistical advice. We express our gratitude to Lisa McCaig for her help with subject recruitment, the dedicated staff of the General Clinical Research Center for subject care and carrying out the study protocol, as well as Dr. Suman Baddam and the Division of Sleep Medicine's Sleep Core staff for polysomnography and EEG analysis support. We thank Drs. Todd Horowitz and Yukiyasu Kamitani for providing a software version of the texture discrimination task.

Correspondence should be addressed to Dr. Daniel Aeschbach, Division of Sleep Medicine, Department of Medicine, Brigham and Women's Hospital, 221 Longwood Avenue, Boston, MA 02115. E-mail: daeschbach@hms.harvard.edu.

DOI:10.1523/JNEUROSCI.5548-07.2008

Copyright $\odot 2008$ Society for Neuroscience $\quad$ 0270-6474/08/282766-07\$15.00/0 mals, sleep can induce synaptic remodeling (Frank et al., 2001), and in humans it has been associated with memory consolidation and learning (for review, see Maquet, 2001; Walker and Stickgold, 2004). Sleep-dependent learning refers to a durable improvement of performance on a given task that occurs slowly and in the absence of continued practice, and only after sleep. Although various aspects of sleep have been associated with learning, slow-wave sleep (SWS) and SWA have specifically been implicated in visuomotor and perceptual skill learning (Gais et al., 2000; Stickgold et al., 2000b; Mednick et al., 2003; Huber et al., 2004). Importantly, practicing a visuomotor task was found to elicit a local increase of SWA that correlated with next-day improvement in task performance (Huber et al., 2004). These and other data (Kattler et al., 1994; Vyazovskiy et al., 2000; Huber et al., 2007) indicate that the generation of SWA is tied to the previous use of a relevant cortical circuit during wakefulness, and directly or indirectly facilitates plastic changes. However, so far the evidence for an active role of SWA in learning is correlative. If SWA is not simply an electrophysiological epiphenomenon, but plays a causal role, interference with its expression should prevent sleep-dependent learning.

Here, we used an acoustic slow-wave suppression paradigm. Our goal was to reduce SWA in NREM sleep by targeted presentation of sounds while avoiding awakenings through careful "titration" of the volume of the sounds. Slow-wave suppression has been used successfully to elucidate homeostatic mechanisms of sleep regulation (Dijk et al., 1987; Gillberg et al., 1991; Ferrara et al., 2002). Here, we tested in humans whether improvement of visual texture discrimination, a form of perceptual learning, depends on SWA. Improvements in texture discrimination occur 
over sleep, are long-lasting, and highly specific to the stimulus characteristics and the retinotopic location, suggesting plasticity at the level of the primary visual cortex (Karni and Sagi, 1993; Schwartz et al., 2002; Walker et al., 2005). We hypothesized (1) that experimental suppression of SWA interferes with sleepdependent improvement in texture discrimination, and (2) that the amount of SWA over the brain area involved in texture discrimination learning is a predictor of the magnitude of such learning.

\section{Materials and Methods}

Subjects. Studies from 16 young (mean age \pm SD, $20.4 \pm 2.9$; range, $18-26$ years), healthy, right-handed subjects (nine women, seven men) are included in the present report. Data from four more study participants were excluded as they did not meet initial performance criteria in the texture discrimination task (TDT) during training (see below). The subjects' health was established by medical history, psychological questionnaires, physical exam, electrocardiography, and comprehensive blood and urine tests. Subjects reported no sleep problems. Before their participation, subjects gave written informed consent to all screening and study procedures.

Conditions and procedures before the study. For the 2 weeks before the study, subjects were instructed to refrain form alcohol, caffeine, nicotine, and any type of drug or medication other than prescribed contraceptives. Compliance was verified during screening by blood and urine toxicology, and again during admission to the study by urine toxicology. For the 2 weeks before the study, subjects were instructed to maintain regular bedtimes and rising times, as well as an $8 \mathrm{~h}$ time in bed, and confirm each bedtime and rising time by calls to a time-stamped voice mail system. Compliance was verified with wrist motor actigraphy (Actiwatch-L; Minimitter, Bend, OR) during 1 week before the study. Women were studied during all phases of their menstrual cycle.

Study protocol. The protocol was approved by the Human Research Committee of Brigham and Women's Hospital. Subjects spent 5 consecutive days in a suite in the General Clinical Research Center at Brigham and Women's Hospital. The protocol included an adaptation (AD) night, a baseline (BL) night, an experimental (EX) night, and a recovery (RC) night. On the $\mathrm{AD}, \mathrm{BL}$ and RC nights, lights-out time corresponded to each subject's habitual bedtime (mean $\pm \mathrm{SD}, 24: 02 \mathrm{~h} \pm 82 \mathrm{~min}$ ) as determined before the study, and time in bed was $8 \mathrm{~h}$ long. On the EX night, lights-out occurred $4 \mathrm{~h}$ later than on the other nights, and time in bed was $4 \mathrm{~h}$ long.

A single-blind parallel-group design was used. Subjects were assigned either to an active group that underwent suppression of EEG SWA $[\mathrm{SWA}(-)$ group; mean age $\pm \mathrm{SD}, 19.7 \pm 2.5$ years; five women, four men] in EX sleep, or a control group $(21.4 \pm 3.3$ years; four women, three men) in which SWA was not suppressed. For the purpose of examining the role of SWA in sleep-dependent learning, we limited EX sleep to $4 \mathrm{~h}$, because previous studies have indicated that (1) the initial part of sleep is particularly important in such learning, and (2) SWA is expressed mainly within the first $4 \mathrm{~h}$ of sleep, after which it levels off. We reasoned that this protocol would maximize the anticipated contrast in learning between the two experimental groups. Subjects were assigned to the two groups in an alternating manner as they completed the screening procedures for the study. Subjects' mean age did not differ between groups (two-tailed $t$ test).

SWA was suppressed with sounds that were triggered from a personal computer in the control room by the study leader (D.A. or A.J.C.), and played through speakers that were placed at both sides of the bed, $\sim 100$ $\mathrm{cm}$ away from the center of the pillow. Each sound consisted of a tone complex of two frequencies $(120$ and $1000 \mathrm{~Hz})$. The sounds started at a volume of $45 \mathrm{dBA}$ and ramped up automatically to a maximum of 100 $\mathrm{dBA}$ (interval between start and maximum, $20 \mathrm{~s}$ ) if not stopped manually by the study leader. The lower frequency of $120 \mathrm{~Hz}$ passed the filter of the digital EEG recorder and was chosen to enable the recording of the sounds via a microphone in the suite that was connected to the EEG recorder. Coregistration of sounds and EEG made it possible to closely examine the impact of the former on the latter. A sound was triggered when the study leader identified a slow wave ( $\leq 2 \mathrm{~Hz}$; no amplitude criterion) in the online EEG recording (C3/A2 derivation) during NREM sleep, and stopped as soon as the slow waves disappeared with the emergence of faster EEG waves. We avoided using a minimal amplitude criterion for slow waves to allow us to reduce EEG SWA maximally. The ramping of the volume made it possible to "titrate" the sounds such to reduce the appearance of slow waves while minimizing awakenings. In some instances, there was a delay of one to several seconds between the onset of the sound and the complete disappearance of slow waves, and in rarer cases it appeared that the sound even triggered a train of a few slow waves. In these cases, the sound was stopped briefly, and a new sound with a steeper ramp of increasing volume was triggered. Thus, whereas the procedure of using sounds was effective in reducing slow waves, it did not result in their complete absence from the EEG.

The study was performed in an environment free of time cues (no windows in suite, no access to clocks, television, radio, telephone, or internet). The light intensity was $\sim 0.23 \mathrm{~W} / \mathrm{m}^{2}(\sim 89 \mathrm{lux}$; measured at 137 $\mathrm{cm}$ from the floor in the horizontal angle). Subjects were continually supervised through closed-circuit television from the control room and through periodic interaction with staff. In addition, during the last $4 \mathrm{~h}$ of the extended wake episode before EX sleep, subjects were accompanied by a staff member to ensure that they maintained wakefulness. During their free time, subjects were allowed to read or write, listen to music, and play card or board games. Movies or video games were not allowed. Subjects were given meals and fluids four times per day, or one time each on the first and last study day.

Polysomnography and EEG analysis. The EEG was recorded during all sleep and wake periods with an ambulatory digital recorder (Vitaport-3; Temec Instruments, Kerkrade, The Netherlands). Recordings were made from all derivations as defined by the international 10/20 system. The EEG electrodes were referenced against the right mastoid (A2). In addition to the EEG, the electrooculogram (EOG), and a two-lead electrocardiogram were recorded, and during the sleep periods the recordings included the submental electromyogram (EMG) as well. Signals were high-pass filtered (time constant, $1.0 \mathrm{~s}$ for EEG and EOG; $0.15 \mathrm{~s}$ for EMG), low-pass filtered (Butterworth, $12 \mathrm{~dB} /$ octave; $-6 \mathrm{~dB}$ at $30 \mathrm{~Hz}$ for EEG and EOG, and at $70 \mathrm{~Hz}$ for EMG), and digitized (resolution, 12 bit; sampling rate, $256 \mathrm{~Hz}$; storage rate, $128 \mathrm{~Hz}$ ). The raw signals were stored on a Flash RAM card (SanDisk, Sunnyvale, CA) and downloaded off-line to a personal computer. Sleep recordings (EEG derivation, C3/A2) were scored by one of us (A.J.C.) on a $30 \mathrm{~s}$ basis according to conventional criteria (Rechtschaffen and Kales, 1968).

The sleep EEG was subjected to off-line spectral analysis. A fastFourier-transform routine (Vitascore; Temec Instruments) was performed on $4 \mathrm{~s}$ epochs ( $10 \%$ cosine-tapered window) resulting in a frequency resolution of $0.25 \mathrm{~Hz}$. Ten consecutive, overlapping $4 \mathrm{~s}$ epochs were averaged into $30 \mathrm{~s}$ epochs after discarding $4 \mathrm{~s}$ epochs that were contaminated by visually identified artifacts (usually because of movements). Data were reduced further by averaging power densities into 0.5 $\mathrm{Hz}$ bins, and discarding spectra $>25 \mathrm{~Hz}$. Finally, mean spectra were matched with the $30 \mathrm{~s}$ sleep scores.

For the purpose of the present study, NREM sleep EEG spectra and SWA are shown for the O2/P4 derivation. This location over the right medial occipitoparietal cortex was chosen, because in the present study the TDT stimuli were presented in the left visual field, and functional magnetic resonance imaging (fMRI) previously mapped overnight TDT learning to this cortical area (Schwartz et al., 2002; Walker et al., 2005).

Learning task. Subjects completed a visual TDT that was adapted from previous studies (Karni and Sagi, 1993; Stickgold et al., 2000b). The subjects' task was to decide whether a small target texture of diagonal bars embedded in an array of horizontal bars was oriented horizontally or vertically. The task was administered during three sessions; a training session on the day after BL sleep, a test session (test 1) after EX sleep, and another test session (test 2) after RC sleep. The sessions began $6 \mathrm{~h}$ (training, test 1 ), or $3 \mathrm{~h}$ (test 2) after rising time.

Subjects were asked to fixate during each trial on a cross in the center of a computer screen (distance from eyes, $\sim 55 \mathrm{~cm}$ ), and when ready press the space bar, after which a sequence of different screens was presented: (1) a blank screen, displayed for 300-400 ms, (2) the target, 
displayed for $14 \mathrm{~ms}$, (3) a blank screen, displayed for an interstimulus interval of 29-500 ms, (4) a mask, displayed for $100 \mathrm{~ms}$ to erase the retinal image of the target display, and (5) another blank screen (no time limit). The target display consisted of an array of $19 \times 19$ horizontal bars spanning $\sim 17^{\circ}$ of visual angle, with the letter " $\mathrm{L}$ " or " $\mathrm{T}$ " in the center, and at a visual angle of $\sim 5^{\circ}$ from the center in the left lower quadrant, three diagonal bars that were aligned either horizontally or vertically. The mask consisted of randomly oriented V-shaped micropatterns and the superimposed letters $\mathrm{L}$ and $\mathrm{T}$ at the center. Subjects were instructed to report by key press at the end of each trial (1) whether the letter in the center of the target display was an L or T, and (2) whether the diagonal bars were aligned horizontally or vertically. The first response served as control for central eye fixation, and subjects were given auditory feedback by the computer if the first response was incorrect. With the second response, the subjects' ability to discriminate visual textures was measured. Only trials with correct letter identification were included in the analysis. The design of the TDT made it possible to systematically increase the visual discrimination difficulty by decreasing the stimulus-to-mask-onset asynchrony (SOA; i.e., interval between the onset of the target display and the mask display).

Each session of the texture discrimination task consisted of a minimum of 1250 trials. Subjects completed the first block of 50 trials in the presence of the study leader. In some subjects one to two extra blocks of 50 trials were added to the training session, if the percentage of correct responses was $<80 \%$ per block at SOAs of 414 or $514 \mathrm{~ms}$. All subjects then completed 24 blocks of 50 trials, with one block each at SOAs of 314 , 257 , and $214 \mathrm{~ms}$, and three blocks each at SOAs of 186, 143, 114, 100, 86, 57 , and $43 \mathrm{~ms}$. Subjects were told that the speed of completing the task was not important. On average, it took $\sim 90 \mathrm{~min}$ to complete a session.

Performance was calculated as the percentage of correct discriminations of horizontal and vertical line orientation at each SOA. Data from four study participants who did not reach a minimum of $80 \%$ correct discriminations at any SOA during training were excluded from analysis. Data from the remaining 16 subjects were plotted as a function of SOA, and each individual's threshold SOA was determined by linear interpolation as the point at which performance dropped below 75\%. Learning was quantified as the decrease in threshold SOA in test 1 and test 2 compared with the training session.

Statistical analysis. The SAS statistical package (versions 6.12 and 9.1.3; SAS Institute, Cary, NC) was used. Each of the sleep parameters derived from polysomnography during EX and RC was subjected to analysis of covariance (ANCOVA) with group [i.e., SWA(-) or control] as a factor, and the BL value for that sleep parameter as a covariate. The mixed model approach was used for the ANCOVA. EEG power densities during EX were normalized for each subject and $0.5 \mathrm{~Hz}$ frequency bin by expressing them as percentages of the mean power densities in the first $4 \mathrm{~h}$ of BL. Log-transformed normalized power densities were compared between groups with two-tailed unpaired $t$ tests.

To investigate the effects of SWA suppression on learning, the differences in the threshold SOA between training and test sessions were analyzed with a repeated measures ANCOVA that was based on the mixed model approach, and included group and test $(1,2)$ as factors, and the absolute threshold SOA during training as a covariate. Because this analysis revealed a significant effect for the covariate $(p=0.01)$, differences between training and test sessions are presented as covariate-adjusted means (see Fig. $2 B$ ), or as percentages of the absolute threshold SOA during training (see Fig. $4 A$ ). Two-tailed paired $t$ tests on the adjusted differences were used to test whether learning has occurred. The magnitude of learning was compared between groups with one-tailed $t$ tests. We used one-tailed tests because our hypothesis was directional, i.e., we had no expectation of finding a larger learning effect after SWA suppression than after control sleep. In the control group, one subject showed deterioration in threshold SOA of $49 \mathrm{~ms}$ in test 1 , and another one an improvement of $92 \mathrm{~ms}$ in test 2. These data points were excluded as outliers as they were beyond the Tukey's inner fences (Tukey, 1977) derived from the interquartile range. Correlation (Pearson) and multiple regression analyses were used to examine the relationship between SWA and other sleep parameters in EX, and the percentage decrease in the threshold SOA on the day after EX.

\section{Results}

Effects of experimental slow-wave suppression on sleep stages and EEG spectra

Presentation of sounds (mean number \pm SD, $151.0 \pm 55.6$ ) during EX sleep resulted in a large reduction of SWS (Table 1). The time spent in SWS was reduced to 26 min (mean \pm SEM, $26.2 \pm$ $7.9 \%$ of the preceding $8 \mathrm{~h}$ BL sleep) in the $\mathrm{SWA}(-)$ group, whereas the control group maintained $74 \mathrm{~min}(89.4 \pm 4.0 \%$ of $\mathrm{BL})$. In contrast, the SWA $(-)$ group spent more time in stage 2 . Waking after sleep onset was slightly higher than in the control group, whereas total sleep time and sleep efficiency were not significantly affected. The amount of REM sleep did not differ between the two groups.

The sounds reduced EEG power density in NREM sleep in the ranges of $0.75-3.0 \mathrm{~Hz}$ and $6.25-6.5 \mathrm{~Hz}$, and increased it in the $13.25-14.0 \mathrm{~Hz}$ range, which is in the frequency range of sleep spindles (Fig. 1). For the purpose of the present study (see Materials and Methods) data are depicted for the O2/P4 derivation, which corresponds to an area over the right medial occipitoparietal cortex. Compared with the first $4 \mathrm{~h}$ of BL, SWA was reduced to $73.0 \pm 7.9 \%$ in the SWA $(-)$ group, whereas the control group showed $102.8 \pm 7.7 \%(p=0.025, t$ test for differences between groups). Similar levels of SWA in response to sounds were found for frontocentral (F3/C3, 70.1 $\pm 7.7 \%$; F4/C4, $72.7 \pm 7.9 \%)$, and left occipitoparietal $(\mathrm{O} 1 / \mathrm{P} 3,72.2 \pm 8.6 \%)$ sites, indicating that the acoustic paradigm resulted in a global reduction of SWA.

Comparisons of the first $4 \mathrm{~h}$ of $\mathrm{BL}$ and $\mathrm{RC}$ sleep showed that in the latter, SWA was increased to $114.1 \pm 5.7 \%$ in the SWA $(-)$ group, whereas the control group exhibited $102.2 \pm 9.7 \%$ ( $p=$ 0.19 for differences between groups). Normalized NREM sleep EEG power spectra that were computed for the first $4 \mathrm{~h}$ as well as for the entire $8 \mathrm{~h}$ of $\mathrm{RC}$ sleep did not differ between groups ( $p>$ $0.05, t$ tests for all $0.5 \mathrm{~Hz}$ bins in the range of $0.25-25.0 \mathrm{~Hz}$ ) (data not shown).

\section{Effects of experimental slow-wave suppression on perceptual learning}

The results of the TDT are summarized in Figure 2. A plot of visual discrimination performance as a function of SOA revealed 


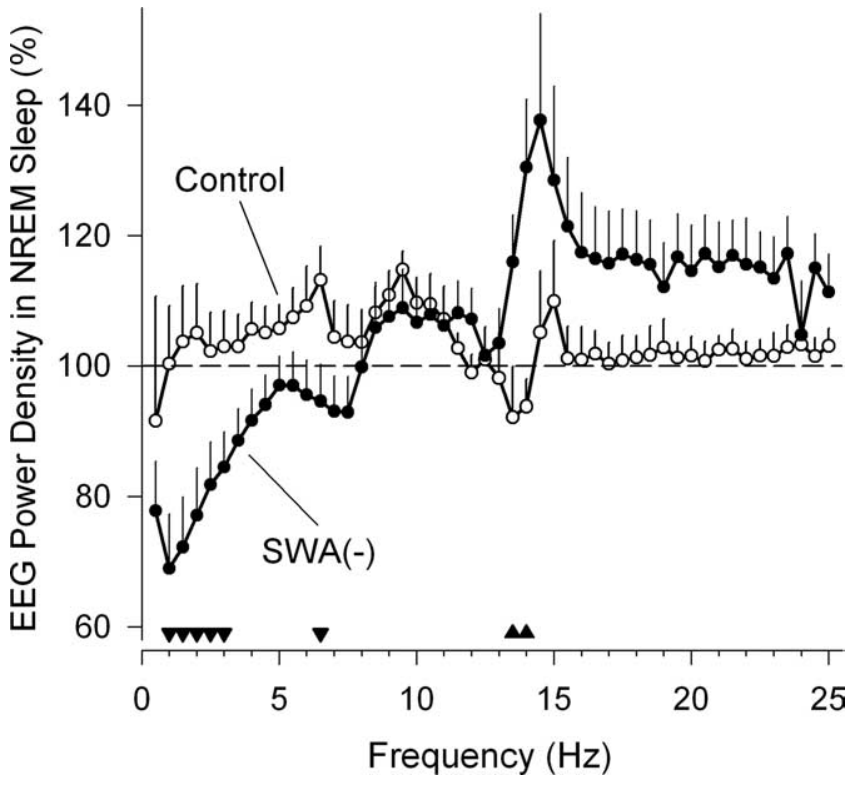

Figure 1. Effect of acoustic slow-wave suppression on EEG power density during NREM sleep over right occipitoparietal cortex (02/P4). Data in the slow-wave activity suppression group, SWA $(-)$, and the control group are expressed as a percentage of power density in first $4 \mathrm{~h}$ of an $8 \mathrm{~h}$ baseline sleep episode. Data represent means + SEM $[n=9$ for SWA $(-)$ and $n=6$ for control, except for frequencies $>15 \mathrm{~Hz}$, for which $n=5$ because of removal of power densities contaminated by high-frequency noise at $\mathrm{P} 4$ in one subject]. Filled triangles above abscissa indicate $0.5 \mathrm{~Hz}$ frequency bins for which differences between groups were significant ( $p<$ 0.05 , unpaired $t$ tests on log-transformed values). Power densities and significance are plotted at upper limit of $0.5 \mathrm{~Hz}$ frequency bins (e.g., value plotted at $1.0 \mathrm{~Hz}$ corresponds to average of power densities at 0.75 and $1.0 \mathrm{~Hz}$ ).

that in both groups and each of the three sessions, performance remained high at long SOAs before declining steeply with decreasing SOAs (Fig. 2 A). Across consecutive sessions, mean performance curves shifted to the left, indicating that subjects were able to maintain high performance at shorter SOAs than in the previous session. This shift corresponds to the effect of learning. To quantify this effect the difference in the threshold SOA between training and test sessions were calculated (Fig. $2 \mathrm{~B}$ ), after adjusting for the influence of the absolute threshold SOA during training on this measure (see Materials and Methods, Statistical analysis). In the control group, the threshold SOA improved in test 1 and test 2 , whereas in the SWA $(-)$ group the improvement was significant only in test 2, i.e., after RD sleep (Fig. 2). Direct comparisons between groups showed that in test 1 the magnitude of improvement was larger in the control than in the SWA $(-)$ group. No significant differences between groups were found for test 2 , or between test 1 of the control group and test 2 of the $\operatorname{SWA}(-)$ group.

To examine whether the effect of slow-wave suppression on performance was indeed attributable to interference with learning and not to a loss of attention, the percentage of correct letter identifications ( $\mathrm{L}$ or $\mathrm{T}$ ) as a measure of visual attention was computed for each of the three TDT sessions (Fig. 3). These percentages did not differ between groups or sessions, and there was no effect of their interaction (two-way repeated measures ANOVA based on mixed model approach: group, $p>0.1$; session, $p>0.5$; group by session, $p>0.8$ ).

\section{Relationship between EEG slow-wave activity and perceptual learning}

Normalized SWA during NREM sleep in EX, and performance improvements in test 1 , i.e., on the day after EX, correlated strongly (Fig. 4A). To examine whether the correlation with performance improvement was specific for SWA, correlation analyses were performed for EEG power densities in all $0.5 \mathrm{~Hz}$ bins within the range of $0.25-25.0 \mathrm{~Hz}$ (Fig. $4 B$ ). This analysis revealed that positive correlations were indeed highest for the lower EEG frequencies $(0.75-6.5 \mathrm{~Hz}$; maximum, 0.75-1.0 Hz). Negative correlations were found within the spindle frequency range $(11.75-12.0 \mathrm{~Hz}, 13.75-14.0 \mathrm{~Hz})$, as well as for most frequencies within the $\beta$ range $(15.75-23.5 \mathrm{~Hz}, 24.25-25.0 \mathrm{~Hz}$ ).

To examine whether the acoustic stimuli per se rather than SWA determined TDT performance, the number of administered sounds during EX sleep was correlated with normalized performance improvement in test 1 . This analysis revealed a marginal positive $(r=0.64 ; p=0.089)$ rather than a negative correlation. The result indicates that the sounds themselves did not prevent performance improvement, but instead that there was a tendency to administer more sounds in subjects in whom they were less effective in interfering with SWA and thereby with performance improvement. Additional evidence for SWA as a determining factor derives from the observation that even within the control group alone performance improvement correlated with SWA in EX sleep $(r=0.91 ; p=0.012)$.

Correlations of performance improvement with total sleep time (Pearson's $r=0.57 ; p=0.028$ ), NREM sleep duration (i.e., stages 2,3 , and $4 ; r=0.39, p=0.15)$, REM sleep duration $(r=$ $0.17 ; p=0.55)$, number of awakenings $(r=-0.49 ; p=0.066)$, EEG $\beta$ activity in NREM sleep (power density in the 16.25-25.0 $\mathrm{Hz}$ range; $r=-0.64, p=0.013)$, and stage 2 sleep $(r=-0.65$; $p=0.008)$ were all lower than the correlation between performance improvement and SWA $(r=0.75 ; p=0.0012)$. Stepwise and backward multiple regression with total sleep time, number of awakenings, stage 2 sleep, $\beta$ activity, and SWA as independent variables ( $\alpha$ level, 0.2 for variable inclusion and retention) revealed that SWA was the only significant predictor of performance improvement.

Finally, there was evidence that SWA after training had an effect on TDT performance not only on the next day, but, to a lesser degree, also on the day after a night of undisturbed RC sleep; SWA in EX correlated marginally with the percentage of TDT performance improvement between training and test 2 [pooled groups, $r=0.45, p=0.091$; control, $r=0.67, p=0.14$; SWA $(-), r=0.27, p=0.45]$.

\section{Discussion}

The present data show that acoustic suppression of SWA in NREM sleep can limit overnight improvement of a visual discrimination skill. Previous studies that implied a role for SWS and SWA in sleep-dependent learning of perceptual and visuomotor skills were based exclusively on correlational evidence (Gais et al., 2000; Stickgold et al., 2000b; Mednick et al., 2003; Huber et al., 2004). The present results indicate that SWA is not simply an electrophysiological epiphenomenon, but that experimental interference with its expression during sleep affects neuronal processes underlying learning directly.

Our study did not include an active control condition involving presentation of sounds during sleep while slow waves were absent; thus, we cannot with absolute certainty exclude the possibility that the acoustic stimulation rather than the suppression of SWA impaired TDT learning. However, we show evidence against this possibility: first, there was no negative correlation between the number of acoustic stimuli and overnight TDT learning, and second, even within the control group, i.e., during 
the complete absence of stimuli, SWA correlated strongly with TDT learning.

It is unlikely that the absence of an improvement in TDT performance after SWA suppression was attributable to compromised attention. Attention was controlled by using a dual task that required correct central letter identification before texture discrimination. Performance on this central task was equally high in the control group and SWA $(-)$ group, and in the latter did not decrease after SWA suppression compared with the training session. Moreover, an fMRI study indicated that TDT learning was associated with increased activation in retinotopic areas of the primary visual cortex (V1) and that this activation was not associated with activation of higher level areas implicated in attentional control (Schwartz et al., 2002). Thus, it is reasonable to conclude that SWA suppression primarily interfered with neuronal processes underlying learning at an early stage of visual processing, whereas acute effects on attention did not play a significant role in TDT performance. This interpretation is consistent with our observation that an individual's SWA level during the first night of sleep after training appeared to affect TDT performance not only on the next day but also after an additional night of (recovery) sleep. Moreover, it was reported previously that improvement in TDT performance over several nights depends in particular on the first night of sleep after training (Stickgold et al., 2000a).

Our data provide evidence for a functional link between neuronal processes of NREM sleep homeostasis and processes underlying learning. This is consistent with other results showing that practicing a visuomotor task induces a local increase of SWA in sleep that correlates with improvement in task performance on the next day (Huber et al., 2004). Remarkably, the increase of SWA $(+27 \%)$ in the initial part of sleep that appeared to facilitate learning in that study, and the reduction of SWA $(-30 \%)$ that limited learning in the present study were of similar magnitude. Previously, Karni et al. (1994) found no effect of SWS deprivation on overnight TDT learning. The seeming discrepancy with the present study may be explained by a difference in the slow-wave content allowed by the experimental protocols. In the previous study, 30 s of SWS (i.e., 1 epoch) needed to be present before an acoustic stimulus was triggered to disrupt further SWS. This procedure undoubtedly must have resulted in longer uninterrupted trains of slow waves in the EEG, and it did result in a larger total amount of SWS $(+15 \%)$ compared with the protocol used here. A more quantitative comparison between the slow-wave content in the different studies is limited by the fact that SWA, which in contrast to SWS is not based on arbitrary definition criteria, has not previously been quantified in TDT paradigms.

Although SWA is the primary marker of sleep homeostasis, analysis of the spectral composition of the NREM sleep EEG and its correlation with overnight TDT performance improvement corroborated the involvement of processes of sleep homeostasis in perceptual learning: the correlation curve (Fig. $4 \mathrm{~B}$ ) with positive coefficients for the lower EEG frequencies and negative co(one-sided unpaired $t$ test).
B
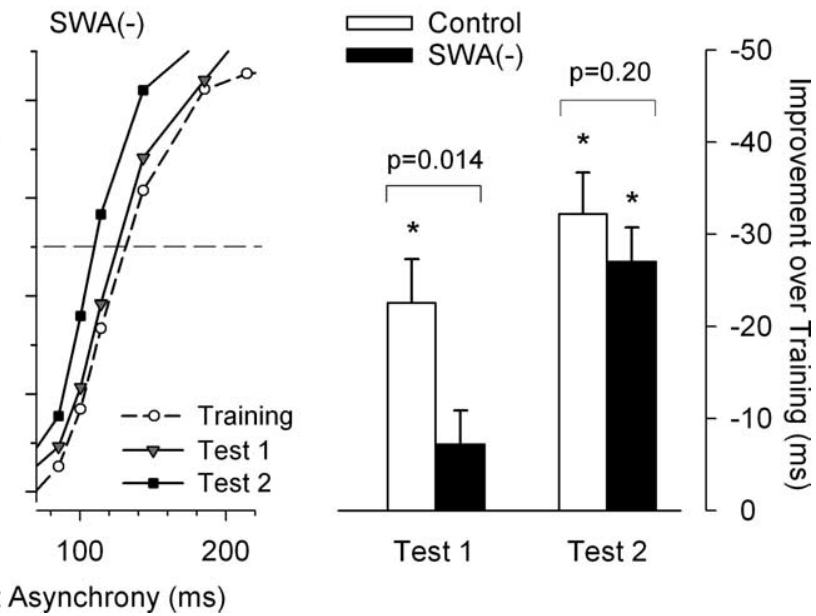

Figure 2. Effects of slow-wave suppression during sleep on learning of a visual discrimination skill. Two groups of subjects .

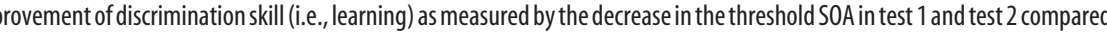
in

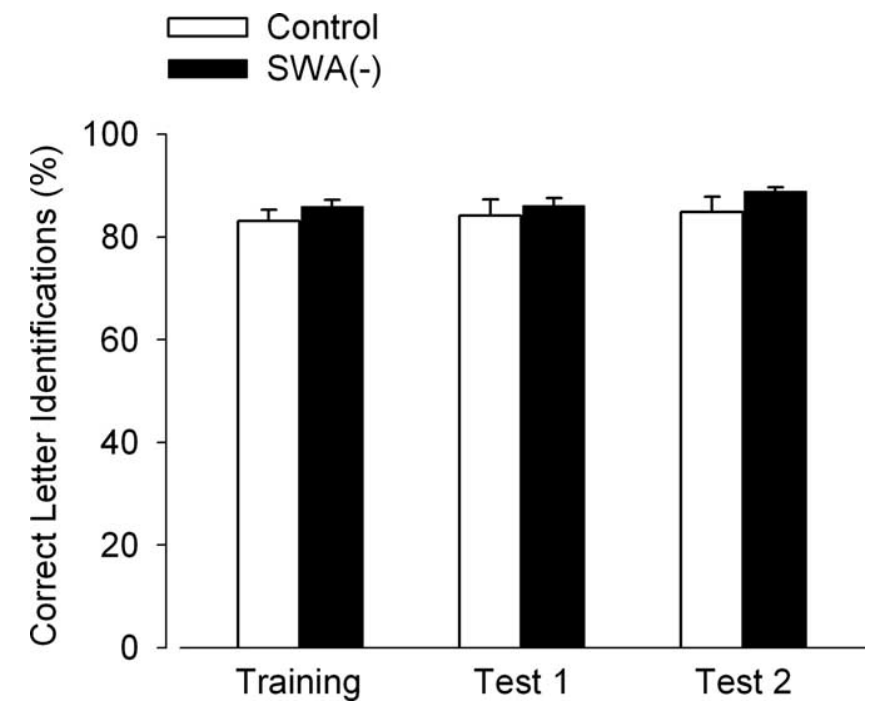

Figure 3. Visual attention in the slow-wave suppression group, SWA(-), and the control group. Attention was measured as the percentage of correct letter identifications ( $L$ or $T$ ) in a dual task that included central letter identification and peripheral texture discrimination. The task was administered during a training session after an $8 \mathrm{~h}$ baseline sleep episode, during test 1 after a $4 \mathrm{~h}$ EX sleep episode, and during test 2 after an $8 \mathrm{~h}$ recovery sleep episode. Slow-wave activity was suppressed during EX sleep. Each session consisted of 1250 trials and lasted $\sim 90$ $\mathrm{min}$. Values represent means $+\mathrm{SEM}$. Attention did not differ between groups or sessions, and there was no interaction between these factors (two-way repeated-measures ANOVA) (see Results).

efficients for the spindle frequency range and the $\beta$ range ( $>16$ $\mathrm{Hz}$ ) is reminiscent of the "EEG spectral signature" of homeostatic sleep pressure, i.e., the pattern of frequency specific changes in the EEG power spectrum that characterize the intensification of 


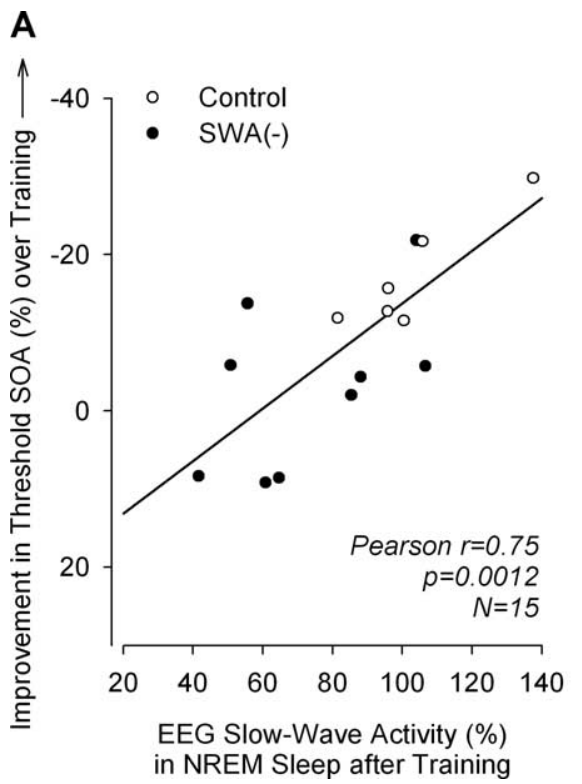

Figure 4. Relationship between EEG power density in NREM sleep and learning of a visual discrimination skill. Learning was quantified as percentage decrease in threshold SOA between a training session (100\%) and a test session completed $24 \mathrm{~h}$ later (Fig. 2). $\boldsymbol{A}$, Linear regression between SWA (power density in the $0.75-4.5 \mathrm{~Hz}$ range) in NREM sleep after training and magnitude of learning. SWA is from 02/P4 derivation. SWA(-) denotes group in which SWA was suppressed with sounds. No sounds were presented in control group. Data are from a $4 \mathrm{~h}$ experimental sleep episode and are expressed as a percentage of SWA in first $4 \mathrm{~h}$ of corresponding baseline sleep. Correlations with learning were similar for SWA from left occipitoparietal derivation $(01 / \mathrm{P} 3, r=$ $0.82, p=0.0002)$ and lower for left frontocentral ( $F 3 / C 3, r=0.64, p=0.010)$ and right frontocentral (F4/C4, $r=0.62, p=$ 0.013 ) derivation (data not shown). Note that the effectiveness of acoustic suppression of SWA varied considerably across individuals, and in two subjects SWA remained close to baseline levels. $\boldsymbol{B}$, Pearson correlations between EEG power density $(02 / \mathrm{P} 4)$ in $0.5 \mathrm{~Hz}$ bins and learning (filled triangles, $p<0.05)$. Because of high-frequency noise in EEG at P4, power densities $>15 \mathrm{~Hz}$ from one subject in control group were excluded from correlation analysis.

NREM sleep in response to sleep deprivation. Such an EEG spectrum is typically characterized by increased power in the lower frequencies with a maximum at $\sim 1 \mathrm{~Hz}$, and reduced power within the spindle frequency and $\beta$ range (Dijk et al., 1990; Aeschbach et al., 1996; Finelli et al., 2001; Achermann et al., 2001). Thus, perceptual learning correlated with all those EEG components that vary as a function of NREM sleep depth. Historically, sleep depth has been defined operationally on the basis of the wake-up threshold, i.e., the difficulty to awaken a sleeping individual (Blake and Gerard, 1937). The current data illustrate that "sleep depth" has broader functional significance in that "deeper" sleep not only protects against awakenings, but also prompts larger gains in behavioral performance.

In the present study, TDT learning did not correlate with the amount of REM sleep. This is consistent with other findings showing that whereas a short sleep episode $(\sim 3 \mathrm{~h})$ rich in SWS benefited TDT learning, an equally long sleep episode rich in REM sleep did not (Gais et al., 2000). Nonetheless, these findings do not rule out the possibility that REM sleep contributes to TDT learning. The latter was suggested in studies in which the sleep episode after TDT training was long enough $(>6 \mathrm{~h})$ to include a sizable amount of REM sleep ( $>80 \mathrm{~min})$ in the latter part of the sleep episode (Karni et al., 1994; Stickgold et al., 2000b). Together, it appears that NREM sleep intensity is the primary determinant of sleep-dependent improvement in TDT performance, whereas the influence of REM sleep is secondary and may depend on the occurrence of SWA early in the sleep episode. Other types of learning have been reported to depend primarily on REM sleep (Smith, 1995; Plihal and Born, 1997; Maquet et al., 2000). In this respect, SWA suppression could serve as an important control paradigm to test whether these other types of learning are indeed unaffected by variations in NREM sleep intensity.

What could be the neuronal processes that link NREM sleep homeostasis to perceptual learning? Sleep has been implicated in neuronal plasticity (Frank et al., 2001), and neuronal activity initiated during waking has been suggested to be reactivated and consolidated during sleep (Wilson and McNaughton, 1994; Peigneux et al., 2004). Yet it appears that genes involved in longterm potentiation, which is an important mechanism of plasticity, are expressed mainly during wakefulness and not during sleep (Cirelli et al., 2004). Tononi and Cirelli (2003, 2006) proposed that whereas synaptic potentiation primarily takes place during waking, SWA in sleep is associated with synaptic downscaling; the latter increases performance on a trained behavioral task through enhancement of the signal-to-noise ratio in a relevant cortical circuit. An important piece in this "synaptic homeostasis hypothesis" is the finding that the slow oscillation in the membrane potential of cortical cells which gives rise to SWA in the EEG occurs at a frequency $(<1$ $\mathrm{Hz}$ ) that in stimulation paradigms was found to be particularly conducive to depotentiation (Kemp and Bashir, 2001) and, thus, to synaptic downscaling. Remarkably, we found that within the frequency range of SWA, the lowest frequencies $(0.75-1.0 \mathrm{~Hz})$ indeed correlated the strongest with overnight improvement in TDT performance. Thus, because these lowest frequencies are likely to reflect more closely the cortical slow oscillation, whereas the higher SWA frequencies may be influenced by oscillatory activity of different origin and functional significance (Steriade et al., 1993), the results of the correlation analysis are consistent with the predictions of the synaptic homeostasis hypothesis.

Previous studies have proposed a role for sleep spindles in some types of learning (Steriade, 1999; Gais et al., 2002; Schmidt et al., 2006; Nishida and Walker, 2007). The fact that in the slowwave suppression protocol we found a negative rather than a positive correlation between TDT performance improvement and spindle frequency activity indicates that the latter does not benefit perceptual learning if it occurs at the expense of SWA. An alternative interpretation, that the induced increase in spindle frequency activity rather than the suppression of SWA limited TDT learning, is theoretically possible but seems less plausible and not supported by previous work. It is known that during much of NREM sleep SWA and spindle frequency activity exhibit opposite time courses (Uchida et al., 1991; Aeschbach and Borbély, 1993). Such an inverse relationship between the two types of activity, which is also evident from their opposite changes in response to sleep deprivation (Borbély et al., 1981), appears to arise from shifts in the membrane potential of thalamic and cortical neurons that characterize changes in NREM sleep depth (Steriade et al., 1993). The present results have important implications in that they caution against interpretations of the role of sleep spindles in learning that do not take into account their interdependence with SWA. 
In conclusion, the present results provide behavioral evidence for a causal role of SWA in overnight improvement of a perceptual skill. The data substantiate the proposed functional link between processes of NREM sleep homeostasis and learning. From a clinical point of view, our results may have implications for conditions such as depression and insomnia, as well as aging, all of which have been associated with reductions in SWA and deficits in learning, two phenomena that may be linked.

\section{References}

Achermann P, Finelli LA, Borbely AA (2001) Unihemispheric enhancement of delta power in human frontal sleep EEG by prolonged wakefulness. Brain Res 913:220-223.

Aeschbach D, Borbély AA (1993) All-night dynamics of the human sleep EEG. J Sleep Res 2:70-81.

Aeschbach D, Cajochen C, Landolt H-P, Borbély AA (1996) Homeostatic sleep regulation in habitual short sleepers and long sleepers. Am J Physiol 270:R41-R53.

Blake H, Gerard RW (1937) Brain potentials during sleep. Am J Physiol 119:692-703.

Borbély AA (1982) A two process model of sleep regulation. Hum Neurobiol 1:195-204.

Borbély AA, Baumann F, Brandeis D, Strauch I, Lehmann D (1981) Sleep deprivation: effect of sleep stages and EEG power density in man. Electroencephalogr Clin Neurophysiol 51:483-493.

Cirelli C, Gutierrez CM, Tononi G (2004) Extensive and divergent effects of sleep and wakefulness on brain gene expression. Neuron 41:35-43.

Daan S, Beersma DGM, Borbély AA (1984) Timing of human sleep: recovery process gated by a circadian pacemaker. Am J Physiol 246:R161-R183.

Dijk DJ, Beersma DGM, Daan S, Bloem GM, van den Hoofdakker RH (1987) Quantitative analysis of the effects of slow wave sleep deprivation during the first $3 \mathrm{~h}$ of sleep on subsequent EEG power density. Eur Arch Psychiatry Neurol Sci 236:323-328.

Dijk DJ, Brunner DP, Borbély AA (1990) Time course of EEG power density during long sleep in humans. Am J Physiol 258:R650-R661.

Ferrara M, De Gennaro L, Curcio G, Cristiani R, Corvasce C, Bertini M (2002) Regional differences of the human sleep electroencephalogram in response to selective slow-wave sleep deprivation. Cereb Cortex 12:737-748

Finelli LA, Borbely AA, Achermann P (2001) Functional topography of the human nonREM sleep electroencephalogram. Eur J Neurosci 13:2282-2290.

Frank MG, Issa NP, Stryker MP (2001) Sleep enhances plasticity in the developing visual cortex. Neuron 30:275-287.

Gais S, Plihal W, Wagner U, Born J (2000) Early sleep triggers memory for early visual discrimination skills. Nat Neurosci 3:1335-1339.

Gais S, Mölle M, Helms K, Born J (2002) Learning-dependent increases in sleep spindle density. J Neurosci 22:6830-6834.

Gillberg M, Anderzen I, Åkerstedt T (1991) Recovery within day-time sleep after slow wave sleep suppression. Electroencephalogr Clin Neurophysiol $78: 267-273$

Huber R, Ghilardi MF, Massimini M, Tononi G (2004) Local sleep and learning. Nature 430:78-81.

Huber R, Esser SK, Ferrarelli F, Massimini M, Peterson MJ, Tononi G (2007) TMS-induced cortical potentiation during wakefulness locally increases slow wave activity during sleep. PLoS ONE 2:e276.

Karni A, Sagi D (1993) The time course of learning a visual skill. Nature 365:250-252

Karni A, Tanne D, Rubenstein BS, Askenasy JJM, Sagi D (1994) Dependence on REM sleep of overnight improvement of a perceptual skill. Science 265:679-682.

Kattler H, Dijk DJ, Borbély AA (1994) Effect of unilateral somatosensory stimulation prior to sleep on the sleep EEG in humans. J Sleep Res 3:159-164.

Kemp N, Bashir ZI (2001) Long-term depression: a cascade of induction and expression mechanisms. Prog Neurobiol 65:339-365.

Maquet P (2001) The role of sleep in learning and memory. Science 294:1048-1052.

Maquet P, Laureys S, Peigneux P, Fuchs S, Petiau C, Phillips C, Aerts J, Del Fiore G, Degueldre C, Meulemans T, Luxen A, Franck G, Van Der LM, Smith C, Cleeremans A (2000) Experience-dependent changes in cerebral activation during human REM sleep. Nat Neurosci 3:831-836.

Mednick S, Nakayama K, Stickgold R (2003) Sleep-dependent learning: a nap is as good as a night. Nat Neurosci 6:697-698.

Nishida M, Walker MP (2007) Daytime naps, motor memory consolidation and regionally specific sleep spindles. PLoS ONE 2:e341.

Peigneux P, Laureys S, Fuchs S, Collette F, Perrin F, Reggers J, Phillips C, Degueldre C, Del Fiore G, Aerts J, Luxen A, Maquet P (2004) Are spatial memories strengthened in the human hippocampus during slow wave sleep? Neuron 44:535-545.

Plihal W, Born J (1997) Effects of early and late nocturnal sleep on declarative and procedural memory. J Cogn Neurosci 9:534-547.

Rechtschaffen A, Kales A (1968) A manual of standardized terminology, techniques and scoring system for sleep stages of human Subjects. Washington, DC: U.S. Government Printing Office.

Schmidt C, Peigneux P, Muto V, Schenkel M, Knoblauch V, Munch M, de Quervain DJ, Wirz-Justice A, Cajochen C (2006) Encoding difficulty promotes postlearning changes in sleep spindle activity during napping. J Neurosci 26:8976-8982.

Schwartz S, Maquet P, Frith C (2002) Neural correlates of perceptual learning: a functional MRI study of visual texture discrimination. Proc Natl Acad Sci USA 99:17137-17142.

Smith C (1995) Sleep states and memory processes. Behav Brain Res 69:137-145.

Steriade M (1999) Coherent oscillations and short-term plasticity in corticothalamic networks. Trends Neurosci 22:337-345.

Steriade M, McCormick DA, Sejnowski TJ (1993) Thalamocortical oscillations in the sleeping and aroused brain. Science 262:679-685.

Stickgold R, James L, Hobson JA (2000a) Visual discrimination learning requires sleep after training. Nat Neurosci 3:1237-1238.

Stickgold R, Whidbee D, Schirmer B, Patel V, Hobson JA (2000b) Visual discrimination task improvement: a multi-step process occuring during sleep. J Cogn Neurosci 12:246-254.

Tononi G, Cirelli C (2003) Sleep and synaptic homeostasis: a hypothesis. Brain Res Bull 62:143-150.

Tononi G, Cirelli C (2006) Sleep function and synaptic homeostasis. Sleep Med Rev 10:49-62.

Tukey JW (1977) Exploratory data analysis. Reading, MA: Addison-Wesley. Uchida S, Maloney T, March JD, Azari R, Feinberg I (1991) Sigma (12$15 \mathrm{~Hz})$ and delta $(0.3-3 \mathrm{~Hz})$ EEG oscillate reciprocally within NREM sleep. Brain Res Bull 27:93-96.

Vyazovskiy V, Borbely AA, Tobler I (2000) Unilateral vibrissae stimulation during waking induces interhemispheric EEG asymmetry during subsequent sleep in the rat. J Sleep Res 9:367-371.

Walker MP, Stickgold R (2004) Sleep-dependent learning and memory consolidation. Neuron 44:121-133.

Walker MP, Stickgold R, Jolesz FA, Yoo SS (2005) The functional anatomy of sleep-dependent visual skill learning. Cereb Cortex 15:1666-1675.

Wilson MA, McNaughton BL (1994) Reactivation of hippocampal ensemble memories during sleep. Science 265:676-679. 